${ }^{1}$ Hospital Metropolitano, Servicio de Salud Metropolitano Occidente. Santiago, Chile. ${ }^{2}$ Hospital San Luis de Buin. Santiago, Chile.

${ }^{3}$ Laboratorio de Carcinogénesis Química y Farmacogenética, Departamento de Oncología Básico Clínica, Facultad de Medicina, Universidad de Chile. Santiago, Chile. ${ }^{4}$ Escuela de Bioquímica Facultad de Ciencias de la Vida, Universidad Andrés Bello. Santiago, Chile.

${ }^{5}$ Servicio de Salud Metropolitano Occidente. Santiago, Chile. ${ }^{6}$ Hospital San Juan de Dios. Santiago, Chile.

${ }^{7}$ Departamento de Ciencias y Tecnología Farmacéutica, Facultad de Ciencias Químicas y Farmacéuticas, Universidad de Chile. Santiago, Chile. aQuímico-Farmacéutico. bBioquímico(a), Ph.D. 'Bioquímico, M.Sc.

Trabajo no recibió financiamiento. Los autores declaran no tener conflictos de interés.

Recibido el 5 de agosto de 2020, aceptado el 20 de enero de 2021.

Correspondencia a: Luis A. Quiñones Laboratorio de Carcinogénesis Química y Farmacogenética, Departamento de Oncología Básico Clínica, Facultad de Medicina, Universidad de Chile.

P.O. Box 70111. Carlos Schachtebeck 299, Quinta Normal. Santiago, Chile. lquinone@med.uchile.cl

Ángela Roco Servicio de Salud Metropolitano Occidente, Santiago de Chile, Av. Alameda Bernardo O'Higgins 2429, Santiago, Chile. angela.roco@redsalud.gov.cl

\section{Impacto de la atención farmacéutica en la calidad del tratamiento con acenocumarol en pacientes con fibrilación auricular}

\author{
BRYAN LEAL ${ }^{1, \mathrm{a}}$, HÉCTOR TORRES ${ }^{2, \mathrm{a}}$, \\ ÁNGELA ROCO ${ }^{3,4,5, b}$, RICARDO ROMÁN ${ }^{2}$, MARIO ROJO $^{3, c}$, \\ ELENA NIETO ${ }^{6}$, LUIS QUIÑONES 3 ,7,b
}

\section{Effects of pharmaceutical counseling on the effectiveness of anticoagulation in patients with atrial fibrillation}

Background: Vitamin Kantagonists such as acenocoumarol and warfarin are usually indicated for the treatment of Atrial Fibrillation (AF). The Therapeutic Range Time (TRT) is a quality of treatment indicator. Values greater than $65 \%$ are associated with significantly lower stroke and bleeding rates. Proper pharmaceutical care improves TRT. Aim: To evaluate the impact of pharmaceutical care in patients with AF treated with acenocoumarol. Material and Methods: We studied 41 patients using acenocoumarol for $A F$ aged $71 \pm 11$ years ( $43 \%$ women). They received pharmaceutical counseling during 12 weeks. TRT was calculated retrospectively for the year before counseling and prospectively during the intervention period. Results: After receiving pharmaceutical counseling TRT improved from $29 \%$ at baseline to $46 \%$ at the end of the intervention $(p<0.01)$. After pharmaceutical care, the adherence of patients to drug treatment improved from $27 \%$ at baseline to $85 \%$ at the end of the study. The user satisfaction survey of the pharmaceutical care received showed a high degree of patient satisfaction. Conclusions: Pharmaceutical care in patients with oral anticoagulant treatment improves TRT of anticoagulation. It is accepted and positively evaluated by patients.

(Rev Med Chile 2021; 149: 724-732)

Key words: Acenocoumarol; Anticoagulants; Atrial Fibrillation; Pharmaceutical Services.

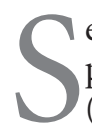
e estima que el $1 \%$ de la población de un país está en tratamiento anticoagulante oral (TACO), con un aumento anual de $10 \%$, asociado a menor mortalidad y aumento de la expectativa de vida ${ }^{1}$. La Fibrilación auricular (FA) es la arritmia cardíaca más común en la práctica clínica, en constante aumento, con 1,6\% en Latinoamérica en la población general y $8,3 \%$ en pacientes sobre los 80 años, $75 \%$ de casos observados en mayores de 60 años y se asocia con alta morbimortalidad ${ }^{1-3}$.

En Chile, para la prevención primaria o secundaria de enfermedad tromboembólica se utilizan los fármacos antagonistas de vitamina K (AVK), siendo el más utilizado Acenocumarol ${ }^{4,5}$, el cual tiene un estrecho margen terapéutico. Algunos alimentos, fármacos y la genética del paciente 
afectan la respuesta farmacológica, lo que explica la necesidad de controlar periódicamente a estos pacientes para ajuste de dosis de $\mathrm{AVK}^{6-10}$.

En el ajuste de la dosis total semanal de AVK el médico utiliza el tiempo de protrombina (expresado como INR) para ajustar la dosis en cada control hasta alcanzar el rango terapéutico esperado $^{8-10}$. Para evaluar la calidad de la anticoagulación se utiliza como indicador el Tiempo en Rango Terapéutico (TRT) el cual debe ser mayor a $65 \%$ para disminuir el riesgo trombótico o hemorrágico ${ }^{9-11}$. En Chile, son escasos los estudios que caracterizan a los pacientes que están bajo tratamiento con AVK. El estudio GARFIELD AF realizado el 2017 en pacientes chilenos con FA mostró una mediana del TRT de $40 \%$ y $3,6 \%$ de Accidente Cerebro-Vascular (ACV) ${ }^{4}$. Otro estudio realizado por el Servicio de Salud Metropolitano Occidente (SSMOc) en 2017, mostró una mediana de $50 \%$ para el TRT en pacientes con FA y un promedio de 301,6 días días para llegar al rango terapéutico deseado ${ }^{5}$.

Por otra parte, en el año 2016 se observó un aumento del consumo de fármacos en los adultos mayores destacándose que $17,3 \%$ de ellos consumen más de 6 fármacos (polifarmacia), lo que conlleva a mayor riesgo de presentar reacciones adversas e interacciones farmacológicas ${ }^{12}$. Una de las soluciones implementadas para abordar esta problemática a nivel mundial es la implementación de un sistema de atención farmacéutica, esto es "la provisión responsable del tratamiento farmacológico, con el propósito de alcanzar resultados concretos que mejoren la calidad de vida del paciente" para asegurar una farmacoterapia apropiada, segura y efectiva ${ }^{2,13,14}$. Varios estudios recientes han demostrado un importante aporte de la atención farmacéutica en el tratamiento anticoagulante oral con AVK, aumentando el \%INR en rango terapéutico y el TRT ${ }^{2,13-23}$.

El objetivo de este estudio es evaluar el impacto de la atención farmacéutica en pacientes con diagnóstico de FA y baja calidad de anticoagulación en tratamiento con acenocumarol que se atienden en el HSLB.

\section{Material y Método}

\section{Aspectos éticos}

Este estudio fue autorizado por el Comité de ética del Servicio de Salud Metropolitano Sur
(Memorando 465/2019 de fecha 31 de mayo de 2019) y por el Comité de ética de la Universidad Nacional Andrés Bello, Facultad de Medicina (Carta s/ $\mathrm{N}^{\circ}$ de fecha junio 2019).

\section{Estudio}

Diseño cuasi-experimental (pre test-post test), involucrando a los mismos pacientes seleccionados para el estudio prospectivo, antes que comenzaran la asesoría farmacéutica; y un estudio descriptivo-prospectivo con asesoría farmacéutica de 12 semanas de duración (01 de julio de 2019 al 30 de septiembre de 2019).

\section{Reclutamiento de pacientes}

En el Hospital San Luis de Buin (HSLB) hay 608 pacientes en tratamiento anticoagulante oral (49\% con fibrilación auricular [FA]) con un TRT promedio de $38,8 \%$.

Luego de citar a un total de 120 pacientes elegibles, según criterios de selección y el análisis de sus fichas clínicas, el médico tratante seleccionó los 52 pacientes enrolados, quienes aceptaron voluntariamente participar. Se incluyeron solo pacientes con diagnóstico de FA del Policlínico de TACO del Hospital San Luis de Buin (HSLB), mayores de 18 años, de ambos sexos y con TRT $<50 \%$, basado en los últimos tres valores de INR. Se excluyeron pacientes con disfunciones hepáticas y/o renales, alcoholismo y uso de otro anticoagulante. Ningún paciente había tenido previamente asesoría farmacéutica. Estos pacientes fueron derivados al Químico farmacéutico para realizar la atención farmacéutica.

\section{Fase retrospectiva}

El tiempo en el rango terapéutico se evaluó durante un período de 1 año, antes del inicio de la fase prospectiva. Para calcular TRT retrospectivo del paciente los datos de INR se obtuvieron desde la ficha clínica electrónica TrakCare-SIDRA. En la fase retrospectiva se utilizó el valor del TRT del último trimestre para compararlos con el TRT al final del período con asesoría farmacéutica.

\section{Fase prospectiva}

Durante el período de estudio, por cada cita del médico tratante, los pacientes también recibieron la atención en box por un químico farmacéutico, en visitas individuales. En la visita 0 el médico seleccionó el paciente elegible según los criterios de inclusión. Posteriormente, el químico farma- 
céutico explicó a los pacientes en qué consistía la asesoría farmacéutica para luego solicitar su participación mediante la firma del consentimiento informado. Durante la asesoría farmacéutica (30 min) se aplicaron cuestionarios, con variables tales como comorbilidades, eventos adversos anteriores, cantidad de ingesta de verduras verdes, medicamentos y otros. El químico farmacéutico enseñó a fraccionar y a utilizar el pastillero semanal. Durante la asesoría farmacéutica, se evaluaron Problemas Relacionados con Medicamentos $(\mathrm{PRM})^{24}$, Reacciones adversas a medicamentos $(\text { RAM })^{25}$, interacciones farmacológicas con alimentos o hierbas ${ }^{26,27} \mathrm{y}$ adherencia al tratamiento con el cuestionario SMAQ (Simplified Medication Adherence Questionnaire), consistente en 6 preguntas que evalúan diferentes facetas del tratamiento: olvidos, rutina, efectos adversos y cuantificación de omisiones ${ }^{28}$. En cada visita, el químico farmacéutico verificó la adherencia mediante el conteo de los comprimidos de acenocumarol (Acebron ${ }^{\circledR}$ ), control de pastilleros y cajas. Se analizaron también las reacciones adversas a medicamentos y PRM a través de encuestas y entrevista clínica. Los PRM se clasificaron según temporalidad pudiendo ser presentes o potenciales y a través de la clasificación de Cipolle y Strand ${ }^{29}$. La causalidad de las RAM se evaluó a través del Algoritmo de Naranjo ${ }^{25}$. Pacientes con valores de INR $<1,5$ y mayores a 3,5 fueron evaluados por el médico considerando la sugerencia del farmacéutico. Durante todas las asesorías farmacéuticas se recolectó y registró la información necesaria para detectar PRM, RAM, interacciones farmacológicas, consideraciones alimenticias, polifarmacia ${ }^{30,31}$ y en forma general el conocimiento del paciente respecto a su farmacoterapia. De ser necesario se efectuó registro fotográfico de manifestaciones de RAM, fraccionamiento del medicamento y metodología de almacenamiento (pastillero) previa autorización escrita del paciente. Los pacientes tuvieron un mínimo de 3 y un máximo de 6 atenciones farmacéuticas. Se realizaron intervenciones educativas personalizadas sobre terapia anticoagulante para sobrellevar y prevenir PRM y RAM. No hubo rotación del personal médico durante el estudio.

\section{TRT e \% INR en rango terapéutico}

TRT se calculó mediante interpolación lineal de valores INR sucesivos ${ }^{32}$. Por su parte el \%INR en rango terapéutico se calculó con el número de INR dentro de rango adecuado en período de tiempo/Número total de INR determinados en ese período) $\mathrm{x} 100^{5}$.

El estudio se realizó mediante comparaciones pareadas, es decir, el TRT de los pacientes se comparó antes y después del tratamiento con acenocumarol con asesoría farmacéutica, de manera que antes de recibir orientación farmacéutica los pacientes recibieron el tratamiento tradicional con acenocumarol por médicos, el grupo de estudio incluyó solo pacientes FA con dosis inestables y que presentaban un TRT $<50$.

\section{Encuesta evaluación atención farmacéutica}

Se evaluó a través de una encuesta realizada a los pacientes por el personal de la oficina de información, reclamos y sugerencias (OIRS) del HSLB.

\section{Análisis estadístico}

Para el análisis se utilizó la media y desviación estándar de los indicadores de calidad (\%INR en rango terapéutico y TRT) en ambos grupos. Se revisó la normalidad a través de Shapiro test. Los análisis se realizaron a través de t-test con un nivel de significancia de $\mathrm{p} \leq 0,05$, en el software STATA 12.0. Una decsripción más detallada puede observarse en (Leal, 2020) ${ }^{33}$.

\section{Resultados}

En la Figura 1 se muestra el diagrama de reclutamiento de este estudio. Un paciente falleció por motivos ajenos a la farmacoterapia (falla renal).

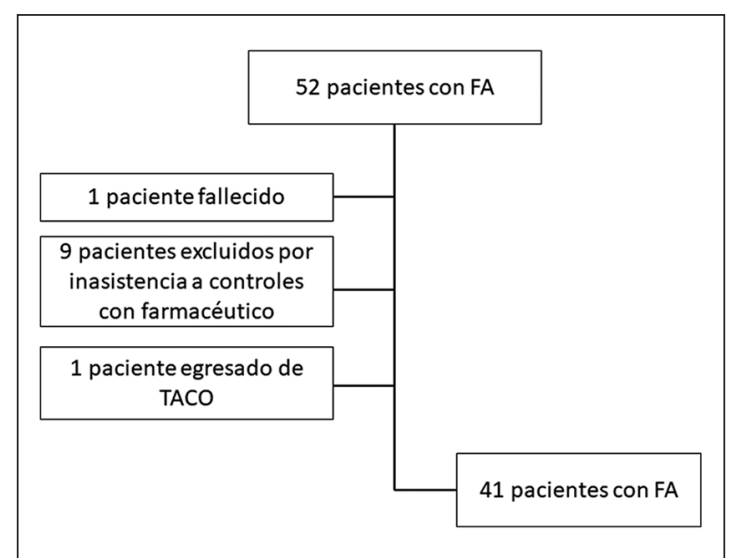

Figura 1. Diagrama de reclutamiento y selección de pacientes. 
En la Tabla 1 se muestran las características demográficas de los pacientes con sus intervenciones y RAM previas a la intervención y en la Tabla 2 se muestran los resultados de los indicadores de calidad del TACO donde se compara los resultados basales (fase retrospectiva) con los resultados al final de la fase prospectiva (3 meses). Para el indicador TRT en la fase retrospectiva el $29,03 \%$ los pacientes se encontraban dentro de rango y al final de la fase prospectiva se observó una mejoría del TRT alcanzando un promedio de 46,13 (p-value 0,0007). El indicador \%INR en rango terapéutico fue de $17,75 \%$ al inicio (fase retrospectiva) y aumentó a $36,5 \%$ al final de la intervención (p-value de 0,0006).

De los pacientes que participaron en el estu- dio $56,1 \%$ tomaba cinco o más medicamentos de manera crónica lo que estableció el perfil de polifarmacia, de acuerdo a los criterios establecidos, es decir "el uso concurrente de muchos fármacos diferentes" o "el uso excesivo de medicamentos" ${ }^{30,31}$. Se detectaron 45 interacciones; siendo las más relevantes con hierbas medicinales (12 casos) y alteraciones en la absorción/distribución debido a las comidas (21 casos).

Durante el estudio se detectaron un total de 13 sospechas de RAM, de las cuales 8 se clasificaron como probable y 5 se clasificaron como posibles según el algoritmo de Naranjo ${ }^{25}$. Las reacciones adversas de mayor frecuencia fueron los sangrados menores, manifestados como hematomas y equimosis. De las reacciones adversas encontradas 3 se

Tabla 1. Características de los pacientes en este estudio y las intervenciones realizadas

\begin{tabular}{|c|c|}
\hline Características & n $(\%)$ \\
\hline Número de pacientes & 41 \\
\hline Promedio Edad \pm DE (años) & $70,83 \pm 11,23$ \\
\hline Índice de masa corporal $(\mathrm{IMC})\left(\mathrm{Kg} / \mathrm{m}^{2}\right) \pm \mathrm{DE}$ & $30,47 \pm 5,39$ \\
\hline Fibrilación auricular & 41 \\
\hline Mujeres, n (\%) & $18(43,9 \%)$ \\
\hline Promedio Edad \pm DE (años) & $74,67 \pm 11,19$ \\
\hline Hombres, n (\%) & $23(56,1 \%)$ \\
\hline Promedio Edad \pm DE (años) & $67,82 \pm 11,37$ \\
\hline \multicolumn{2}{|l|}{ Comorbilidades* } \\
\hline HTA & 30 \\
\hline Insuficiencia cardiaca congestiva (ICC) & 11 \\
\hline Diabetes mellitus tipo II & 19 \\
\hline Dislipidemias & 21 \\
\hline Accidente cerebro vascular (ACV) & 10 \\
\hline Hipotiroidismo & 17 \\
\hline Enfermedad pulmonar obstructiva crónica (EPOC) & 2 \\
\hline Enfermedad renal crónica & 1 \\
\hline Asma & 1 \\
\hline Antecedente de sangrado previo a la consulta** & 14 \\
\hline$N^{\circ}$ de RAM previas (no sangrado)** & 13 \\
\hline \multicolumn{2}{|l|}{ Intervenciones farmacéuticas } \\
\hline$N^{\circ}$ de Evaluaciones de Adherencia & 84 \\
\hline No de Pautas de administración de Medicamentos realizadas & 28 \\
\hline No intervenciones educativas con material impreso (Trípticos, tablas, etc.) & 135 \\
\hline
\end{tabular}

DE: desviación estándar. HTA: hipertensión arterial. *En general cada paciente presentó alrededor de 3 comorbilidades, en promedio. ${ }^{* *}$ Objetivadas en ficha clínica y/o referido por paciente. 
Tabla 2. Resultados del impacto de la atención farmacéutica en los indicadores de calidad de tratamiento TRT y \% INR en rango terapéutico

\begin{tabular}{|llc|}
\hline Indicadores & Resultados & p-value \\
\hline Tiempo en rango terapéutico (TRT) & & 0,0007 \\
$\quad$ Promedio al inicio \pm DE & $29,03 \pm 22,85$ & $46,13 \pm 30,92$ \\
$\quad$ Promedio al finalizar \pm DE & & 0,0006 \\
\% INR en rango terapéutico & $17,75 \pm 16,47$ & \\
$\quad$ Promedio al inicio \pm DE & $36,50 \pm 29,70$ & \\
\hline
\end{tabular}

$p<0,05$ es considerado significativo.

asocian a interacciones farmacológicas: a) Diarrea por lactulosa + laxante natural; b) Taquicardia y visión borrosa en paciente usuario de doble beta bloqueo y c) hematuria asociada a ketoprofeno 50 $\mathrm{mg} \mathrm{c} / 8 \mathrm{~h}$ por 9 días + tratamiento con acenocumarol según esquema.

Se encontraron un total de 57 PRM a lo largo del estudio, 29 de Cumplimiento, 1 de Seguridad, 23 de Eficacia y 4 de Indicación.

Se realizaron mediciones de adherencia al inicio y al final de la intervención a todos los pacientes utilizando el cuestionario $\mathrm{SMAQ}^{28}$, el resultado de la evaluación de adherencia en la primera entrevista mostró que el 26,8\% de los pacientes era adherente al tratamiento farmacológico, el que mejoró con la atención farmacéutica a $85,4 \%$ al final de la fase prospectiva.

En la Figura 2 se muestran ejemplos de errores detectados en la partición de los comprimidos en cuartos, medios y tres cuartos (Figura 2a), y el mal uso de los pastilleros, donde en lugar de colocar la dosis diaria, colocan en cada casillero los distintos fármacos que utilizan (Figura 2b), lo que fue corregido durante la atención farmacéutica.

En la Tabla 4 se muestran los resultados de la encuesta satisfacción usuaria aplicada en la última atención por farmacéutico (tercer mes) y que fue aplicada por personal de OIRS del HSLB.

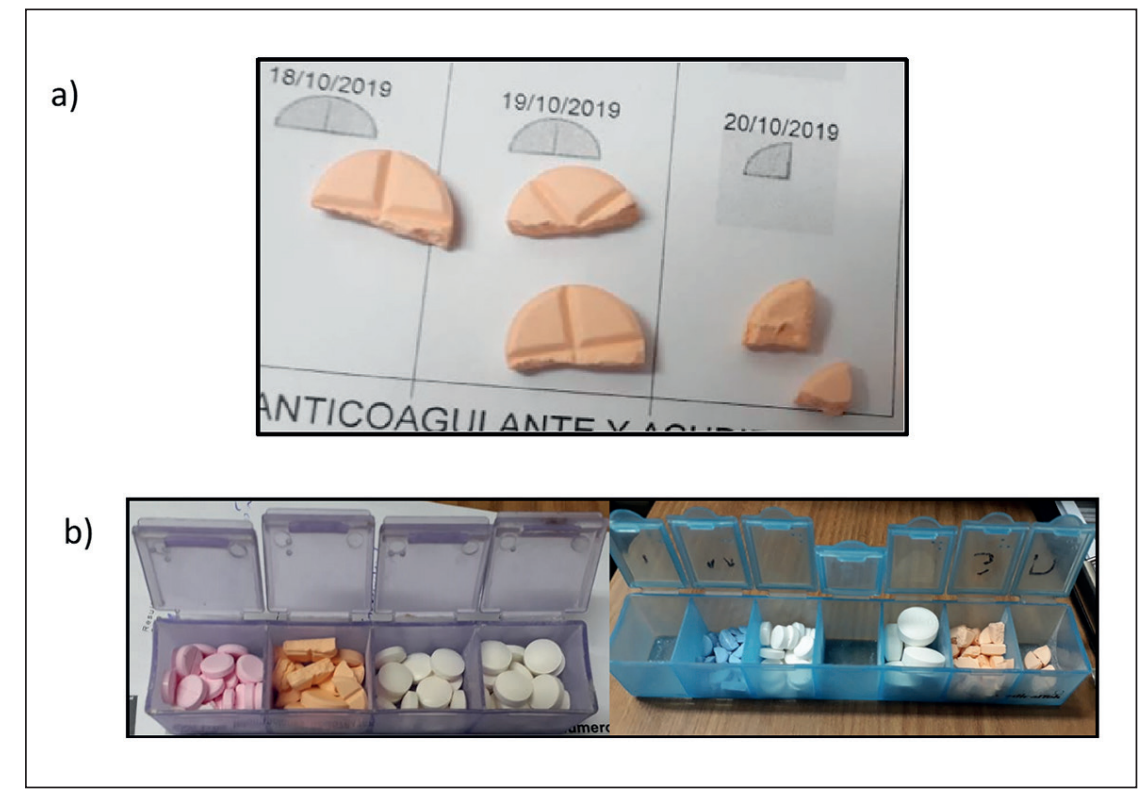

Figura 2. Errores detectados en el grupo sin atención farmacéutica, a) ejemplos de errores partición comprimidos de acenocumarol: se observa diversos cortes asimétricos de los comprimidos, con clara variabilidad de las dosificaciones. b) ejemplos de errores en el uso de 2 pastilleros, utilizados para almacenar otros medicamentos. 
Tabla 3. Interacciones detectadas en la asesoría farmacéutica

\begin{tabular}{|c|c|c|c|c|c|}
\hline \multicolumn{6}{|c|}{ Interacciones totales detectadas } \\
\hline $\begin{array}{l}\text { Potenciales } \\
\text { (riesgo) }\end{array}$ & $\begin{array}{l}12 \text { casos } \\
\text { Acenocumarol/ } \\
\text { Hierbas medicinales }\end{array}$ & $\begin{array}{l}2 \text { casos } \\
\text { Acenocumarol } \\
\text { Levotiroxina }\end{array}$ & $\begin{array}{l}1 \text { caso } \\
\text { Acenocumarol/ } \\
\text { Ácido acetil salicílico }\end{array}$ & $\begin{array}{l}2 \text { casos } \\
\text { Acenocumarol } \\
\text { Amiodarona }\end{array}$ & $\begin{array}{l}2 \text { casos } \\
\text { Acenocumarol/ } \\
\text { Omeprazol }\end{array}$ \\
\hline Presentes & \multicolumn{5}{|c|}{$\begin{array}{l}\text { - } 21 \text { Casos acenocumarol con alimentos (almuerzo y/u once) } \\
\text { - } 2 \text { Casos levotiroxina con alimentos } \\
\text { - } 1 \text { Caso acenocumarol-ketoprofeno } \\
\text { - } 1 \text { Caso lactulosa-laxante natural }\end{array}$} \\
\hline
\end{tabular}

Tabla 4. Resultados encuesta satisfacción usuaria en pacientes con asesoría farmacéutica

\begin{tabular}{|c|c|c|c|}
\hline Evaluación del Programa de Atención Farmacéutica & $\begin{array}{c}\text { Muy } \\
\text { Recomendable } \\
\text { (n) }\end{array}$ & $\begin{array}{l}\text { Medianamente } \\
\text { Recomendable } \\
\text { (n) }\end{array}$ & $\begin{array}{c}\text { No } \\
\text { Recomendable } \\
\text { (n) }\end{array}$ \\
\hline $\begin{array}{l}\text { ¿Recomendaría a sus familiares o amistades que acudan a un Quí- } \\
\text { mico Farmacéutico para obtener este tipo de servicio? }\end{array}$ & 41 & - & - \\
\hline $\begin{array}{l}\text { ¿Recomendaría que este servicio otorgado por el Químico Farma- } \\
\text { céutico pueda ser implementado para otros Programas de Salud? }\end{array}$ & 41 & - & - \\
\hline $\begin{array}{l}\text { ¿Recomendaría este Servicio porque considera que le ayudó a } \\
\text { mejorar su calidad de vida? }\end{array}$ & 41 & - & - \\
\hline
\end{tabular}

\section{Discusión}

En este estudio, los indicadores TRT y \%INR en rango terapéutico mostraron una mejoría estadísticamente significativa entre el inicio y el término del estudio (p-value 0,0007 y 0,0006 , respectivamente). En este aspecto, un estudio realizado en Brasil en pacientes con FA en tratamiento con warfarina mostró que el promedio de TRT con asesoría farmacéutica por un año aumenta respecto del valor basal' ${ }^{2}$. Así también, varios estudios de tipo prospectivo han evaluado el impacto de la asesoría farmacéutica en conjunto con la atención médica, mostrando excelentes resultados ${ }^{13-17,21-23,31}$.

La mejoría observada del TRT se adjudicó a mejor adherencia al tratamiento por la educación realizada además de la implementación de estrategias personalizadas de adherencia, de manera personalizada, ya que muchos pacientes no sabían por qué estaban en TACO y los riesgos involucrados.

Se sugirió al médico tratante eliminar el fraccionamiento de $3 / 4$ debido a la dificultad detectada en los pacientes para lograr una buena partición de los comprimidos (Figura 2). Los pacientes evaluaron muy bien la asesoría farmacéutica (Tabla 3 ).

La mayor cantidad de PRM detectados correspondió a 29 de cumplimiento y 23 de eficacia según la clasificación de Cipolle y Strand. La cantidad de PRM detectados de este tipo es considerablemente mayor a los PRM detectados de tipo Seguridad e Indicación. Esto está estrechamente relacionado para el caso de los PRM de cumplimiento con los antecedentes de la baja adherencia inicial, y la presencia de polifarmacia en los pacientes. Estos hallazgos concuerdan con diversos estudios en donde se observa que la polifarmacia impacta negativamente en la adherencia de los pacientes, aumentando el riesgo de fracaso terapéutico y eventos adversos, además del aumente en el número de $\mathrm{PRM}^{34-36}$. Por otro lado, la falta de conocimiento sobre la terapia farmacológica, explica la presencia de 23 casos de problemas de Eficacia (causados todos por interacciones farmacológicas), ya que estos pacientes por comodidad y desconocimiento tomaban Acenocumarol junto a sus demás medicamentos, o la administración con alimentos, lo que puede afectar y volver errática 
la absorción afectando los valores de INR y por lo tanto afectando también los valores de INR en rango terapéutico y $\mathrm{TRT}^{37,38}$.

Las pautas de administración de medicamentos elaboradas promovieron la adherencia y ayudaron a que los pacientes recordaran la terapia farmacológica prescrita, además entregar un horario de administración adaptado a la rutina y quehacer del paciente, reduciendo interacciones y ayudando a disminuir los PRM. Esto explicaría el descenso observado en el número de PRM detectados a medida que transcurrió este estudio, desde 31 PRM el primer mes, 21 PMR el segundo mes, llegando hasta 5 PRM al finalizar el tercer mes de atención farmacéutica, lo cual también se observa en otros estudios realizados en diversos niveles asistenciales y con distintos perfiles farmacológicos como en el caso de pacientes pluripatológicos, VIH positivo, enfermedad renal crónica, etc. En todos los ejemplos mencionados, se observa una disminución del número de interacciones y $\mathrm{PRM}^{39-41 .}$

Este es el primer estudio en Chile que evalúa el impacto de la Asesoría Farmacéutica en la calidad del tratamiento anticoagulante con AVK y podría ser extrapolable a otros pacientes en terapia anticoagulante debido a los múltiples factores que pueden afectar la seguridad y eficacia del tratamiento con AVK (interacciones, cambios en la alimentación, etc.) y lo crítico de la falta de adherencia en todos los tratamientos crónicos. Sin perjuicio de lo anterior, este trabajo presenta algunas limitaciones que es relevante mencionar. El bajo tamaño muestral ancanzado luego de la aplicación de los criterios de selección no permite realizar análisis de subgrupos, evaluando por ejemplo la influencia de obesidad, sexo, etnia o edad. Finalmente, el tiempo de seguimiento de solo tres meses podría enmascarar algunos resultados relevantes observables a más largo plazo. Por lo tanto, un estudio a más largo plazo y con poblaciones más grandes de estudio es altamente recomendable.

En conclusión, la Asesoría Farmacéutica en pacientes TACO realizada por profesionales comprometidos, permite mejorar los indicadores de calidad de TACO, mejorando la adherencia al tratamiento lo que impacta en un menor número de eventos adversos y disminución del gasto en salud pública. La Asesoría Farmacéutica acerca al especialista a pacientes complejos y es aceptada, valorada y muy bien calificada por los pacientes.
Agradecimientos: Al equipo Directivo del Hospital San Luis de Buin que dio las facilidades para la realización de este estudio y a los pacientes por aceptar participar en este estudio.

\section{Referencias}

1. Boned-Ombuena A, Pérez-Panadés J, López-Maside A, Miralles-Espí M, Guardiola Vilarroig S, Adam Ruiz D, et al. Prevalencia de la anticoagulación oral y calidad de su seguimiento en el ámbito de la atención primaria: estudio de la Red Centinela Sanitaria de la Comunitat Valenciana. Aten Primaria. 2017; 49 (9): 534-48. Spanish. doi: 10.1016/j.aprim.2016.11.015. Epub 2017 Apr 10. PMID: 28408116; PMCID: PMC6876010.

2. Marcatto LR, Sacilotto L, Tavares LC, Facin M, Olivetti N, Strunz CMC, et al. Pharmaceutical Care Increases Time in Therapeutic Range of Patients With Poor Quality of Anticoagulation With Warfarin. Front Pharmacol. 2018; 9: 1052. doi: 10.3389/fphar.2018.01052. PMID: 30298004; PMCID: PMC6160801.

3. Barcellona D, Mastino D, Marongiu F. Portable coagulometer for vitamin K-antagonist monitoring: the patients' point of view. Patient Prefer Adherence. 2018; 12: 1521-6. doi: 10.2147/PPA.S164680. PMID: 30197503; PMCID: PMC6112808.

4. Corbalán R, Conejeros C, Rey C, Stockins B, Eggers G, Astudillo C, et al. Features, management and prognosis of Chilean patients with non-valvular atrial fibrillation: GARFIELD AF registry. Rev Med Chile 2017; 145 (8): 963-71. https://dx.doi.org/10.4067/s003498872017000800963.

5. Nieto E, Suárez M, Roco Á, Rubilar JC, Tamayo F, Rojo M, et al. Anticoagulation Management With Coumarinic Drugs in Chilean Patients. Clinical and Applied Thrombosis/Hemostasis 2019, 25. https://doi. org/10.1177/1076029619834342.

6. Bosch J, Eikelboom JW, Connolly SJ, Bruns NC, Lanius V, Yuan F, et al. Rationale, Design and Baseline Characteristics of Participants in the Cardiovascular Outcomes for People Using Anticoagulation Strategies (COMPASS) Trial. Can J Cardiol 2017; 33(8): 1027-35.

7. Esteve-Pastor MA, Rivera-Caravaca JM, Roldán-Rabadán I, Roldán V, Muñiz J, Raña-Míguez P, et al. Quality of oral anticoagulation with vitamin $\mathrm{K}$ antagonists in 'real-world' patients with atrial fibrillation: a report from the prospective multicentre FANTASIIA registry, EP Europace 2018; 20 (9): 1435-41, https://doi. org/10.1093/europace/eux314.

8. Leite PM, Martins MAP, Castilho RO. Review on me- 
chanisms and interactions in concomitant use of herbs and warfarin therapy. Biomed Pharmacother 2016; 83: 14-21. doi: 10.1016/j.biopha.2016.06.012. Epub 2016 Jun 17.

9. Guyatt GH, Akl EA, Crowther M, Gutterman DD, Schünemann HJ. Executive summary: Antithrombotic therapy and prevention of thrombosis, 9th ed: American College of Chest Physicians evidence-based clinical practice guidelines. Chest 2012; 141 (2 SUPPL.): 7-47.

10. Ageno W, Gallus AS, Wittkowsky A, Crowther M, Hylek EM, Palareti G. Oral anticoagulant therapy: Antithrombotic Therapy and Prevention of Thrombosis, 9th ed: American College of Chest Physicians Evidence-Based Clinical Practice Guidelines, Chest 2012; 141: e44S-88.

11. NICE, Atrial fibrillation: the management of atrial fibrillation (CG180), Issued: June 2014, last modified: August 2014. Disponible en: https://www,nice,org,uk/ guidance/cg180.

12. Urriola R. Cambio demográfico y envejecimiento: ¿está preparado el sistema de salud?. Boletín Semestral Economía y Salud 2019; 13( 1): 11-8.

13. de Lima Silva RG, Bertollo CM, Ferreira IG, Brant LC, Martins MAP. Assessment of oral anticoagulation control at two pharmacist-managed clinics in Brazil. Int J Clin Pharm 2017; 39 1157-61. 10.1007/s11096-0170511-X

14. Hepler C, Strand 1. Opportunities and responsabilities in pharmaceutical care. Am J Hosp Pharm 1990; 47 (3): 533-43.

15. Choumane NS, Malaeb DN, Malaeb B, Hallit S. A multicenter, prospective study evaluating the impact of the clinical pharmacist-physician counselling on warfarin therapy management in Lebanon. BMC Health Serv. Res. 2018; 18: 80. 10.1186/s12913-018-2874-7.

16. Verret L, Couturier J, Rozon A, Saudrais-Janecek S, St-Onge A, Nguyen A, et al. Impact of a pharmacist-led warfarin self-management program on quality of life and anticoagulation control: a randomized trial. Pharmacotherapy 2012; 32: 871-9. 10.1002/j.18759114.2012.01116.

17. Katada Y, Nakagawa S, Minakata K, Odaka M, Taue H, Sato Y, et al. Efficacy of protocol-based pharmacotherapy management on anticoagulation with warfarin for patients with cardiovascular surgery. J Clin Pharm Ther 2017; 42: 591-7. 10.1111/jcpt.12560.

18. Lee T, Davis E, Kielly J. Clinical impact of a pharmacist-led inpatient anticoagulation service: a review of the literature. Integr. Pharm Res Prac 2016; 5: 53-63. doi: 10.2147/IPRP.S93312.

19. Aidit S, Soh YC, Yap CS, Khan TM, Neoh CF, Shaharuddin S, et al. Effect of standardized warfarin treatment protocol on anticoagulant effect: comparison of a warfarin medication therapy adherence clinic with usual medical care. Front. Pharmacol. 2017,8: 637. doi: 10.3389/ fphar.2017.

20. Tran N, Lin C, Do N, Muradian I, Lu Q, Henderson S. The Impact of Implementing an Advance Practice Pharmacist-Led Anticoagulation Clinic Within a Correctional Facility. J Pharm Pract. 2019. doi: 10.1177/0897190019892120. [Epub ahead of print].

21. Ohgushi A, Nakayama N, Namiki A, Nagashima A, Ogawa R, Akazawa M, et al. A Retrospective Evaluation of the Impact of Multi-disciplinary Approach for Improving the Quality of Anticoagulation Therapy in Ambulatory Patients with Non-valvular Atrial Fibrillation Receiving Warfarin. Yakugaku Zasshi. 2019; 139 (9): 1177-83. doi: 10.1248/yakushi.18-00209.

22. Mifsud E, Wirth F, Camilleri L, Azzopardi LM, Serracino-Inglott A. Pharmacist-led medicine use review in community pharmacy for patients on warfarin. Int J Clin Pharm 2019; 41 (3): 741-50. doi: 10.1007/s11096019-00824-4. Epub 2019 Apr 22.

23. Mckenzie J, Wilson-Clarke C, Prout J, Campbell J, Douglas R, Gossell-Williams M. Improving warfarin therapy through implementation of a hospital-based pharmacist managed clinic in Jamaica. Pharm Pract 2018; 16 (4): 1214. doi: 10.18549/PharmPract.2018.04.1214. Epub 2018 Nov 7. PMID: 30637024; PMCID: PMC6322982.

24. van Mil JW, Westerlund LO, Hersberger KE, Schaefer MA. Drug-related problem classification systems. Ann Pharmacother 2004; 38 (5): 859-67.

25. OPS. Organización Panamericana de la Salud (2010). Buenas Prácticas de Farmacovigilancia para las Américas, Grupo de trabajo en Farmacovigilancia, Red PARF, Documento Técnico No 5. Disponible en: https:// www.paho.org/hq/index.php?option=com_docman\&view=download\&category_slug=documentos-8499\&alias $=33513$-buenas-pra-cticas-farmacovigilancia-ame-ricas-2010-513\&Itemid=270\&lang=es.

26. Ge B, Zhang Z, Zuo Z. Updates on the clinical evidenced herb-warfarin interactions. Evidence-based complementary and alternative medicine: eCAM 2014; 957362. doi: 10.1155/2014/957362.

27. Patel JA, Gohil KJ. Warfarin-herb interactions: a review and study based on assessment of clinical case reports in literature. Boletin Latinoamericano y del Caribe de Plantas Medicinales y Aromáticas 2008; 7 (2): 85-99.

28. Ortega F y Grupo de estudio VATREN. et al. Validación del cuestionario simplificado de adherencia a la medicación (SMAQ) en pacientes con trasplante renal en terapia con tacrolimus, Nefrología 2011; 31 (6): 690-6. 
29. Cipolle R, Strand L, Morley P. Pharmaceutical Care Practice: The Clinician's Guide. Segunda Edición. USA, McGraw Hill Medical; 2004.

30. Hovstadius B, Petersson G. Factors leading to excessive polypharmacy. Clin Geriatr Med. 2012; 28 (2): 159-72. doi: 10.1016/j.cger.2012.01.001. Epub 2012 Feb 15. PMID: 22500536

31. Walckiers D, Van der Heyden J, Tafforeau J. Factors associated with excessive polypharmacy in older people. Archives of public health $=$ Archives belges de sante publique 2015; 73: 50. https://doi.org/10.1186/s13690015-0095-7.

32. Rosendaal FR, Cannegieter SC, Van der Meer FJM, Briet E. A method to determine the optimal intensity of oral anticoagulant therapy. Thromb Haemost. 1993; 69 (3): 236-9.

33. Leal B. Impacto de la atención farmacéutica en la calidad del tratamiento anticoagulante oral (TACO) en pacientes con diagnóstico de Fibrilación Auricular que se atienden en el consultorio adosado de especialidad del Hospital San Luis de Buin, Tesis para optar al título de Químico Farmacéutico de la Universidad Andrés Bello. 2020.

34. Ramírez J, Orozco Hernández J, Marín Medina D. Polifarmacia y prescripción de medicamentos potencialmente no apropiados en ancianos. Revista Médica de Risaralda 2015; 21(2): 52-7. https://doi. org/10.22517/25395203.12451.

35. Ascar G. Polifarmacia en la tercera edad Polypharmacy in the elderly. Pharmaceutical Care España 2015; 11(4): 163-8.
36. Gómez Aguirre N, Caudevilla Martínez A, Bellostas Muñoz L, Crespo Avellana M, Velilla Marco J, Díez-Manglano J. Pluripatología, polifarmacia, complejidad terapéutica y uso adecuado de la medicación. Revista Clinica Espanola 2017; 217 (5): 289-95. https://doi. org/10.1016/j.rce.2016.12.013.

37. Lee J, Grace K, Taylor A. Effect of a Pharmacy Care Program on Medication Adherence and Persistence, Blood Pressure, an Low Density Lipoprotein Cholesterol. A Randomized Controlled Trial. JAMA 2006; 296 (21): 2563-71.

38. Monforte HC De. Tratamiento anticoagulante oral. Disponible en: https://www.yumpu.com/es/document/ view/14673428/tratamiento-anticoagulante-oral-fisterra.

39. Delgado-Silveira E, Fernández-Villalba EM, Freire MGM, Pérez MSA, Lagranja MPC, Martí JFP. The impact of Pharmacy Intervention on the treatment of elderly multi-pathological patients. Farmacia Hospitalaria 2015; 39 (4): 192-202. https://doi.org/10.7399/ fh.2015.39.4.8329.

40. San H, Coquimbo P. De. Impacto de la Atención Farmacéutica en la adherencia de los pacientes con VIH en el Hospital San Pablo de Coquimbo, Chile. Pharmaceutical Care Espana 2017; 19 (1): 3-15. https://www.pharmcareesp.com/index.php/PharmaCARE/article/view/369.

41. Sánchez-Gili M, Toro-Chico P, Pérez-Encinas M, Montaña AM, Portolés-Pérez JM. Intervención farmacéutica en la adherencia al tratamiento de pacientes con enfermedad renal crónica. Revista de Calidad Asistencial 2011; 26(3): 146-51. https://doi.org/10.1016/j. cali.2010.11.012. 